\title{
Agôn
}

Revue des arts de la scène

$8 \mid 2019$

Matières

\section{Matières en transformation}

Entretien réalisé par Aurélie Coulon

\section{Élise Vigneron et Aurélie Coulon}

\section{CpenEdition}

Journals

Édition électronique

URL : http://journals.openedition.org/agon/6488

DOI : 10.4000/agon.6488

ISSN : 1961-8581

Éditeur

Association Agôn

Référence électronique

Élise Vigneron et Aurélie Coulon, "Matières en transformation », Agôn [En ligne], 8 | 2019, mis en ligne le 23 mai 2020, consulté le 04 juin 2020. URL : http://journals.openedition.org/agon/6488 ; DOI :

https://doi.org/10.4000/agon.6488

Ce document a été généré automatiquement le 4 juin 2020

Association Agôn et les auteurs des articles 


\title{
Matières en transformation
}

\author{
Entretien réalisé par Aurélie Coulon \\ Élise Vigneron et Aurélie Coulon
}

\section{NOTE DE L'ÉDITEUR}

Entretien réalisé le 09/11/2018 au TJP - CDN Grand Est

1 Élise Vigneron est metteuse en scène, marionnettiste et plasticienne. Formée aux arts plastiques, au théâtre et au cirque, puis diplômée de l'École Nationale Supérieure des Arts de la Marionnette de Charleville-Mézières en 2005, elle a été marionnettiste et scénographe au sein de la compagnie de théâtre d'ombres Le Théâtre de Nuit (Aurélie Morin) et collaboratrice artistique auprès du chorégraphe Gang Peng et de la marionnettiste Aurélie Hubeau.

Elle a créé en 2008 Traversées, spectacle déambulatoire sans parole conçu pour investir des espaces non dédiés à la représentation, librement inspiré de la pièce Seuils de Patrick Kermann. Ce premier spectacle ${ }^{1}$ reposait sur une écriture visuelle proche de l'installation plastique et sur une forte présence des éléments (eau, terre, feu, air). Créé au festival Mondial de Théâtre de Marionnettes à Charleville-Mézières, Traversées a obtenu le prix de la forme innovante au Festival international de marionnettes de Lleida (Espagne) en 2011.

Sa compagnie, le Théâtre de l'Entrouvert ${ }^{2}$, existe depuis 2010. Elle a créé en 2011 le spectacle Traversées/Fragments, forme courte et musicale issue de Traversées. Pour la pièce Impermanence, créée en 2013 en collaboration avec Eleonora Gimenez, équilibriste et danseuse, elle s'est inspirée des textes de l'auteur norvégien Tarjei Vesaas, qui l'ont amenée à travailler avec des matériaux éphémères et instables et à expérimenter avec la glace. Dans Anywhere, pièce créée en 2016 et librement inspirée du roman d'Henri Bauchau $E$ dipe sur la route ${ }^{3}$, elle a poursuivi cette recherche sur la glace de l'état solide à l'état gazeux en mettant en scène une marionnette de glace figurant $\mathbb{E}$ dipe.

Son spectacle, L'Enfant (création 2018, TJP-CDN Grand Est), adapté de La Mort de Tintagiles de Maeterlinck, est une forme immersive dans laquelle le spectateur évolue à travers un espace labyrinthique habité par des scénographies éphémères. 
En 2019 elle a présenté au Festival d'Avignon Axis mundi, co-création avec la breakeuse Anne Nguyen, dans un dispositif plastique évolutif et éphémère fait de glace.

\section{Parcours et formation}

Aurélie coulon: Tu as une formation de plasticienne et tu as ensuite été élève de l'École Nationale Supérieure des Arts de la Marionnette de Charleville. Quelle a été la place de la matière dans ton parcours? A-t-elle été l'un des déclencheurs de ton envie d'aller vers la scène?

Élise VIGNERON : Ce qui m'intéressait dans les arts plastiques, c'était les installations - et j'en ai toujours fait, dès l'enfance. J'aimais bien utiliser des végétaux, de la cire... Je viens de la campagne, j'étais toujours dans la nature et j'ai toujours eu ce lien avec la matière brute, naturelle, et aussi le goût de l'état dans lequel cela me met de faire des installations : on s'immerge, on crée un monde qui a moins de limites, on est dans le sensible, dans les odeurs, les mouvements, les couleurs, le rythme des saisons. Quand j'étais étudiante à Aix j'arrivais toujours en cours avec tout un bazar intransportable que je crée à base de matériaux naturels. Je trouvais que dans l'art contemporain le rapport aux œuvres n'était pas assez vivant, dans le domaine des arts plastiques j'avais besoin d'être en lien avec les spectateurs. Je ne dirais plus la même chose aujourd'hui car il y a maintenant beaucoup de formes immersives en arts plastiques, mais à l'époque les études d'art étaient très conceptuelles. Mes études m'ont permis de mettre au cœur de la réflexion la question de la réception d'une œuvre d'art.

Entre temps j'ai beaucoup pratiqué le cirque; le rapport à la matière y est aussi très important mais c'est différent de la marionnette - c'est très en lien avec le sensible, le corps et la déclinaison de possibilités que cela offre par rapport aux matériaux. Je faisais de l'acrobatie aérienne et surtout du trapèze depuis toute petite puis je suis allée à l'école de Lomme ${ }^{4}$ à Lille. J'ai dû arrêter car je me suis blessée. Dans les arts du cirque il y a vraiment une plongée dans la matière alors qu'en marionnette on passe souvent par la recherche de l'anthropomorphisme.

Les arts de la marionnette me sont alors apparus comme étant le langage qui me correspondait, parce qu'elle réunissait la possibilité d'un langage silencieux avec la matière mais aussi le mouvement. La démarche est moins conceptuelle qu'en arts plastiques, plus sensible. Mon solo Traversés se composait de sept installations. Chaque cadre était habité par des installations en mouvement qui puisaient leur caractère dramatique au sein même de l'organicité de la matière. La terre, l'eau, la poussière, le feu, le sel, le bois sont des éléments vivants. Leurs densité et fluidité entrent en écho avec les sensations intérieures et la mémoire subjective du spectateur.

Ensuite, les poèmes de Tarjei Vesaas m'ont amenée à travailler avec la glace, matériau qui incarne l'idée de transformation. En tant que marionnettiste, je me suis posé ces questions : est-ce qu'on peut entrer en compassion, en empathie avec une matière abstraite comme un écran de glace, sans être face à une forme anthropomorphe ? Estce que le simple fait que la matière se transforme peut nous renvoyer à notre condition humaine d'être éphémère?

\section{Matériaux de travail}

A. C. J'ai l'impression qu'il y a deux fils qui se croisent dans ton travail : d'un côté les objets anthropomorphes, pour lesquels tu collabores souvent avec Arnaud Louski-Pane ou Hélène 
Barreau, et d'un autre côté des matériaux plus diffus. Comment ces deux chemins se sontils mis en place?

É. V. Je pense que c'est lié à ce qui est au fondement de ma démarche. Je travaille toujours à partir d'une idée plastique ou d'une idée de dispositif liée à un concept, ensuite je cherche un texte qui ne va pas me servir de trame narrative mais qui va pouvoir se frotter à cet univers plastique. Je constitue une sorte de corpus par rapport à l'auteur, qui regroupe les archétypes, les motifs récurrents dans son travail. Je m'attache plus aux symboles qu'à l'histoire, travaillant par strates, par sensations, par imbrications. Ce sont des paysages que je crée avec la matière, à l'intérieur il y a des personnages qui sont là comme des figures, des ombres qui les traversent : Tintagiles est l'enfant, étymologiquement "celui qui ne parle pas", c'est un être qui fait le passage entre les mondes et conduit Ygraine, sa sœur, au domaine des ombres. Les marionnettes ont la faculté de parler à travers leur silence et de nous faire sentir ce qui n'est pas dicible.

\section{Anywhere (1)}

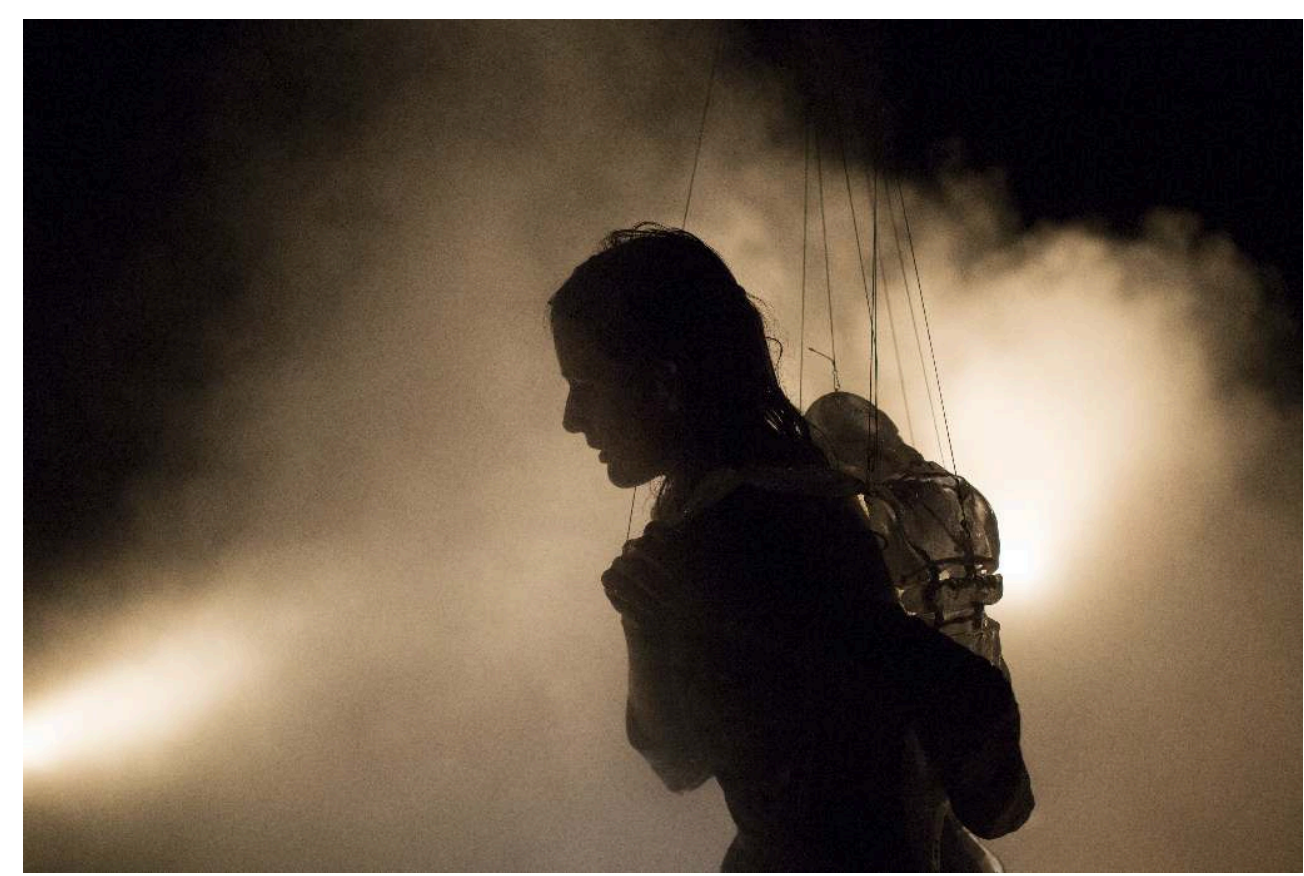

(c) Vincent Beaume

Dans Anywhere c'était différent car tout est en lien avec la fonte du personnage de glace. Ce que va vivre Ædipe se reflète dans la scénographie, créant des paysages qui sont une expansion de ce qu'il vit physiquement. Les écritures à l'encre noire qui coulent au début du spectacle sur l'écran de glace sont un présage à la blessure des yeux d'Æđdipe, qui vont couler noir pendant le spectacle. De même quand ce corps de glace commence à fondre, la pluie envahit l'espace en écho. Dans L'Enfant c'est autre chose, ce spectacle est plutôt un prolongement de mon solo Traversées, qui est une déambulation. Les objets sont assez figuratifs - les pierres, les os, les planches par exemple - mais ils peuvent se moduler. Avec l'équipe (Sarah Lascar, la marionnettiste, Julie Denisse, la comédienne, Arnaud Louski-Pane qui m'a accompagnée sur le dispositif et Manon Worms, la dramaturge), on a fait toute une recherche sur l'idée d'un espace qui se construit en direct, puis se détruit. La scénographie est une métaphore de la réalité 
intérieure du personnage central, représenté par Ygraine. Les différents états que traverse Ygraine tout au long de la pièce, soumission, rébellion, soulèvement, résignation, ouverture se lisent au travers de l'évolution de la scénographie.

\section{L'Enfant (1)}

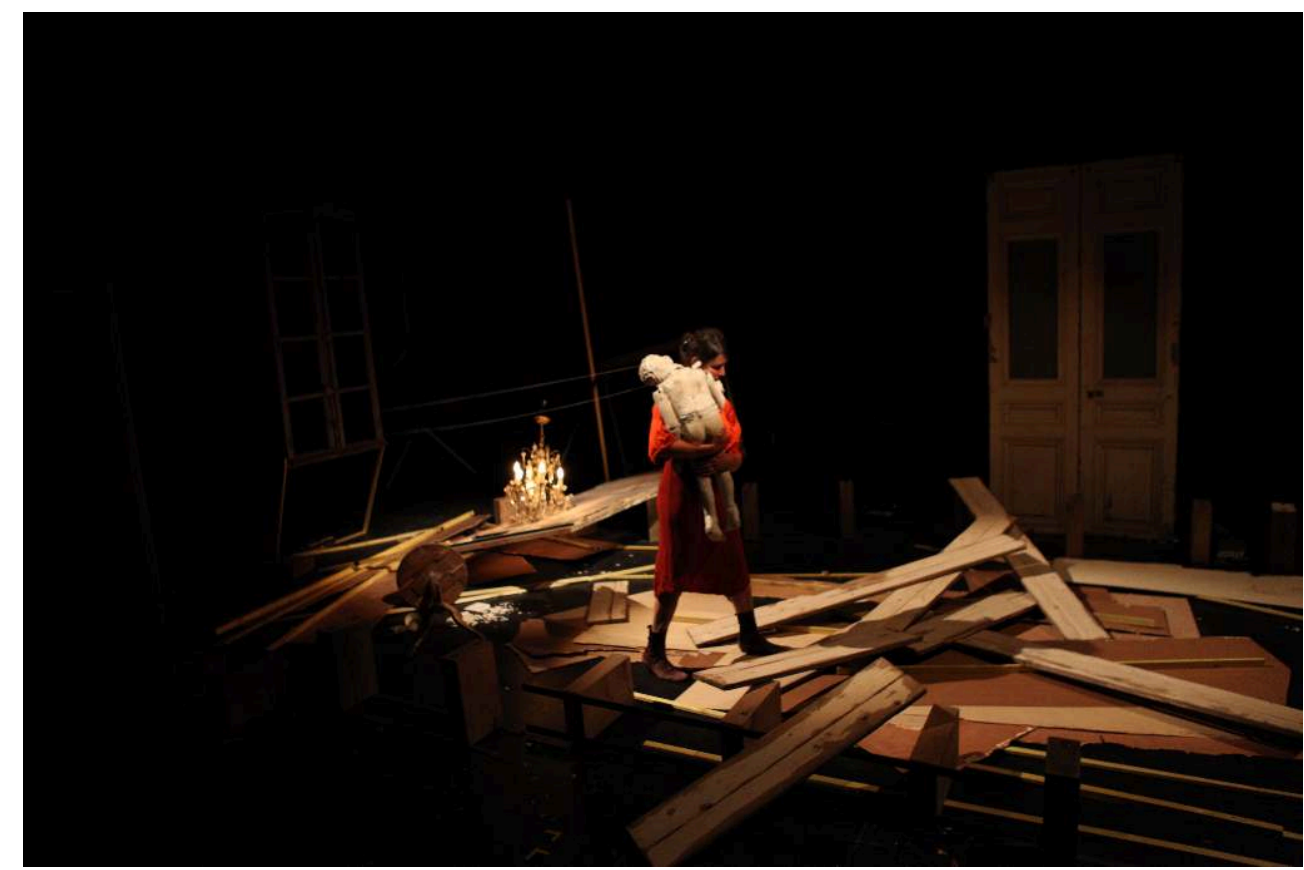

A. C. J'aimerais qu'on parle plus en détail de tes choix dramaturgiques car les textes que tu choisis me paraissent porteurs d'un potentiel scénographique - ou du moins d'enjeux spatiaux. Je pense aux poèmes de Tarjei Vesaas sur lesquels tu t'es appuyée dans Impermanence, mais le roman $\mathbb{E}$ dipe sur la route d'Henry Bauchau raconte aussi des histoires de seuils d'initiation. Qu'est-ce qui t'intéresse dans ces textes ? Est-ce que tu y cherches les trames de parcours, de trajectoires... ?

É. V. En fait j'aime bien l'idée que les matériaux éphémères nous permettent de faire l'expérience du temps cyclique. C'est dans Anywhere que je travaille vraiment làdessus :la matière nous parle de notre propre transformation. L'Enfant aborde davantage les espaces, les interstices, les territoires que l'on ne peut pas forcément exprimer avec la parole, les états de transformation de matière ou de scénographie : les spectateurs sont invités à rentrer dans la pièce de manière physique par les sensations, en éprouvant les vibrations, les tremblements, les choses qui se cassent, les murs qui s'écroulent... C'est tout de suite direct : ça va parler sans passer par le langage verbal. C'est aussi une métaphore du personnage. J'aime mettre en lien ces éléments : le personnage central, la scénographie et la façon dont ça résonne auprès du spectateur.

A. C. Je me demandais si tu te nourris de références plastiques particulières: œuvres, artistes...?

É. V. Oui, beaucoup. Pour La Mort de Tintagiles j'ai beaucoup travaillé sur la notion de sublime. Je pars de points de vue archétypaux, de la figure mythique de la mèreogresse ou de Déméter qui donne et qui reprend vie. Dans le texte il y a un point de vue symboliste unilatéral et j'avais envie de me situer d'un point de vue plus ouvert, plus mythique, où la vie et la mort sont reliées: la mère dévoratrice est aussi une énergie vitale qui pousse à la révolte. J'aime bien travailler sur ces deux pendants, qui 
sont reliés : soumission et soulèvement, visible et invisible... En fait, je travaille tout le temps sur de telles articulations et cela se reflète toujours dans la scénographie. L'idée du sublime était pour moi aussi une manière de penser le rapport avec le monde actuel. Dans La Mort de Tintagiles le monde est stérile, il n'y a plus de nature et c'était ce que je voulais traiter par le minéral. Cela me renvoyait au livre Sublime, les tremblements $d u$ monde $e^{5}$ c'est le catalogue d'une exposition qui a été présentée au Centre Pompidou-Metz il y a quelques années, sur le sublime au XIX ${ }^{\mathrm{e}}$ siècle en tant que manière d'appréhender la grandeur de la nature. Maintenant, dans l'art contemporain, le sublime est aussi le rapport à notre potentielle disparition. Il y a aussi cette dimension dans la pièce : la mère dévoratrice, l'ogresse, peut aussi être la nature qui veut reprendre ses droits. Il y a l'idée du tremblement, de ces catastrophes qui surgissent : la présence de la reine ne se matérialise ici que par des vibrations et relève d'un niveau cosmogonique, plus large que celui des personnages.

A. C. La marionnette de Tintagiles a un visage très singulier et expressif.

É. V. Avec Arnaud Louski-Pane, qui a construit la marionnette, on s'est inspirés de la statuaire grecque : elle n'a pas de pupilles, elle a l'œil creux et je pense que cela joue. La matière a un côté évanescent. Elle a aussi un mouvement et des articulations proches des nôtres, et elle a un tombé dans le poids qui est équilibré comme notre corps. Sa particularité est de pouvoir être manipulée par des fils et aussi comme une marionnette portée.

L'Enfant (2)

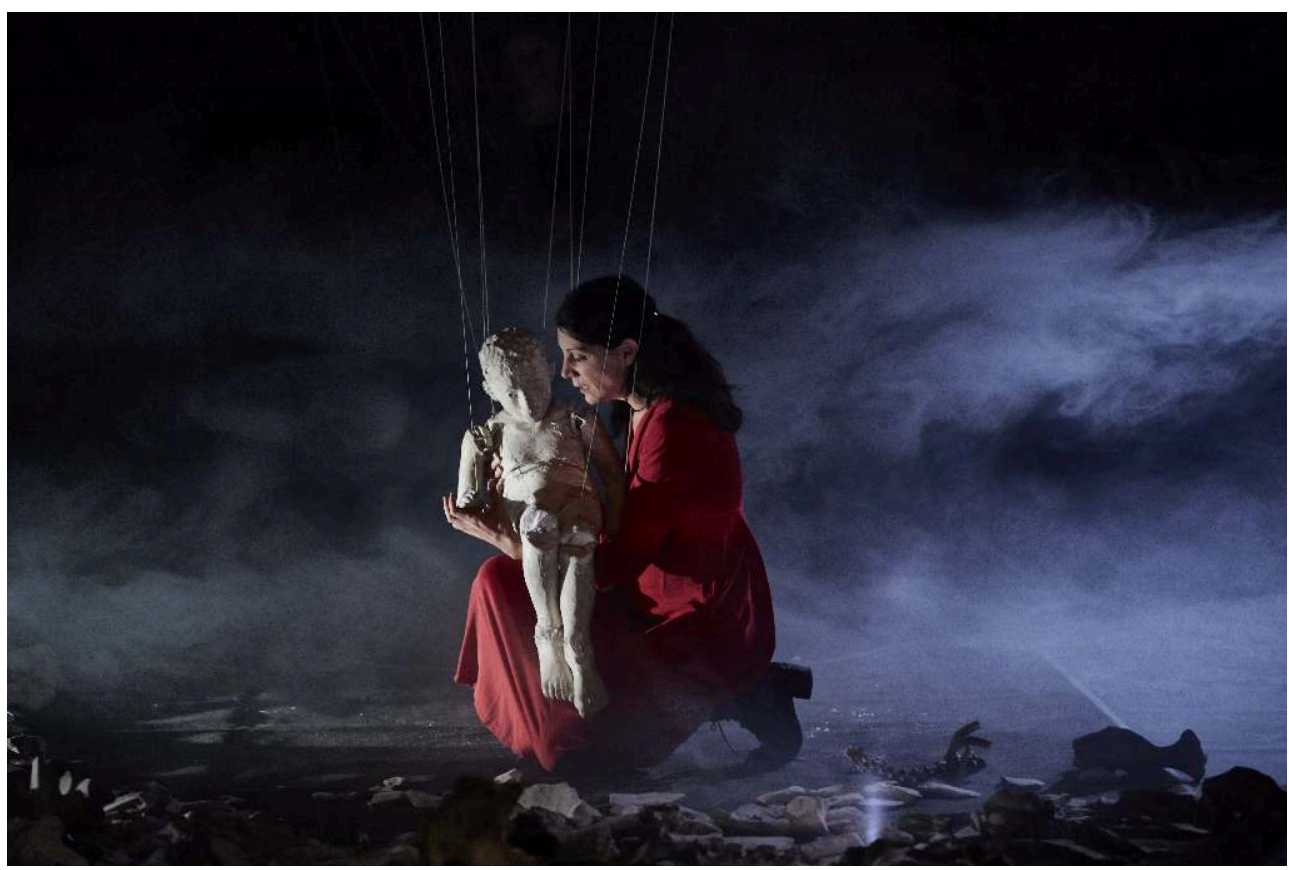

(c) Christophe Loiseau

\section{Animation(s) : l'espace vibrant}


A. C. Est-ce que pour toi cela fait écho au texte d'ouverture de Jérémy Damian pour le dernier numéro de la revue Corps-Objet-Image ${ }^{6}$ ?

É. V. Oui. D’ailleurs, j'avais participé à ce numéro ${ }^{7}$. Je suis très intéressée par ce qu'écrit Jérémy Damian, et la façon dont il met en perspective le travail sur la matière. Cela me fait penser aussi au livre Vivre avec les catastrophes ${ }^{8}$, où l'auteur Yoann Moreau parle des états psychiques qui suivent les catastrophes (répétition, bégaiement, déconstruction du langage), mais aussi des forces vitales qui reviennent après et qui nous dépassent. Nous nous sommes beaucoup inspirés du livre Soulèvements de Georges Didi-Huberman ${ }^{9}$ pour le personnage d'Ygraine. Nous avons adapté la pièce autour de ce personnage central, et nous avons choisi une fin ouverte qui peut être interprétée différemment selon chacun, nous éloignant de la vision très noire présente dans le texte. Cette ouverture vient des images et des matériaux mis en jeu qui créent un écart avec le texte dit.

\section{A. C. C'est un espace très matriciel qui se construit à partir de là.}

É. V. En fait, cela vient de Jung. Il y a quelque temps, j'ai lu un livre de Marie-Louise von Franz, une psychologue jungienne qui travaille beaucoup sur les contes de fées en lien avec la figure de la femme ${ }^{10}$. C'est aussi une structure mythique présente dans les contes : le roi et la reine veulent tuer le petit prince parce que d'un point de vue psychique c'est l'ancien qui reste figé, qui détient le pouvoir, qui est assis, qui limite comme le personnage d'Ygraine, qui se soumet à la raison mais qui du coup se coupe de sa force vitale, de son enfant intérieur pourrait-on dire. L'enfant, ce serait la nouveauté qui essaye d'émerger et la manière dont le psychisme veut garder un équilibre tout en étant travaillé par cette force-là, qui essaye de déstabiliser les choses. On est toujours en train de se construire et de se détruire, finalement. Et c'est ça qui va vraiment déclencher la bascule du personnage.

\section{L'Enfant (3)}

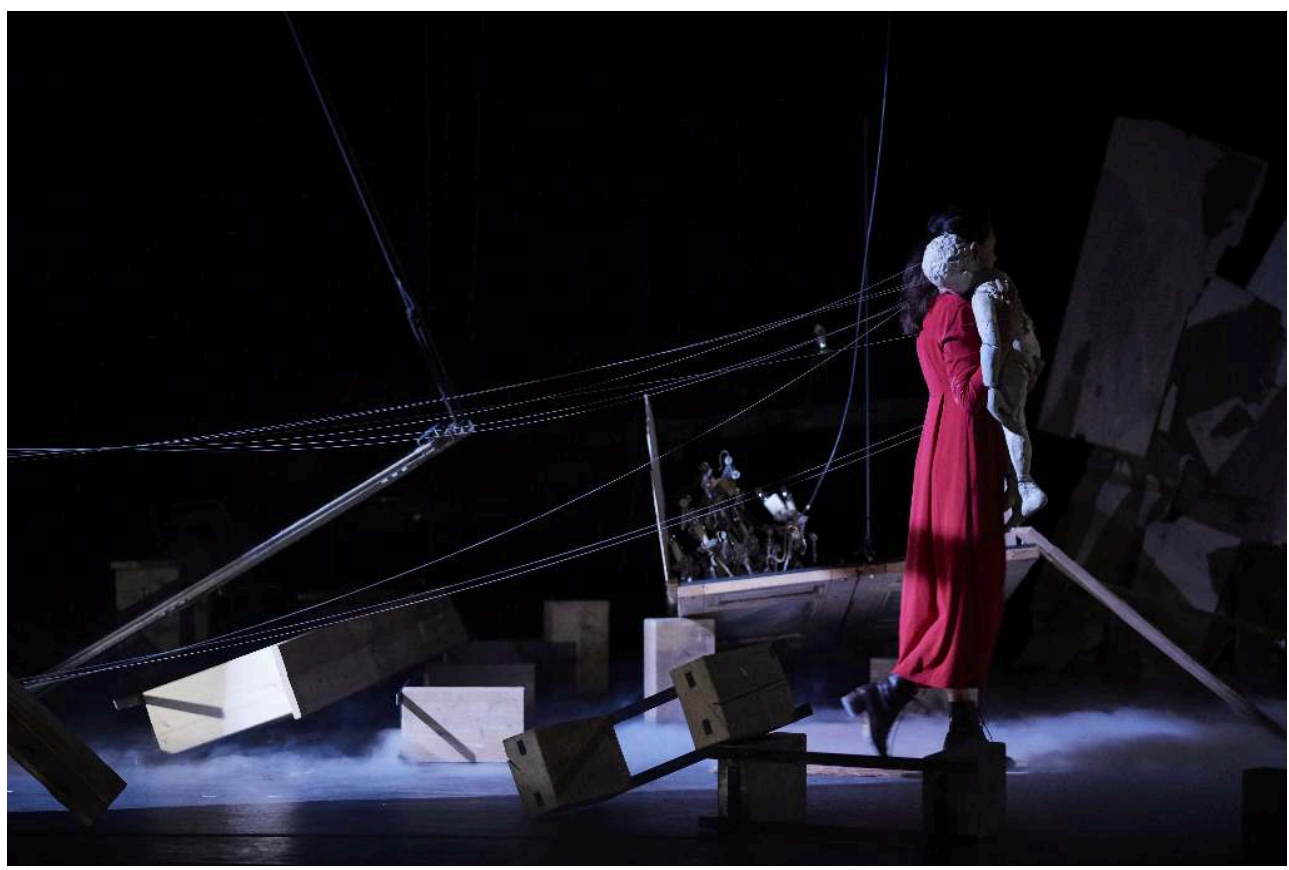

(c) Christophe Loiseau 
A. C. À travers cette dimension cosmogonique et l'importance de la scénographie, il y a quelque chose dans ce spectacle et dans les précédents qui questionne profondément la notion de manipulation, qu'on ne peut limiter à une action de la main. J'ai l'impression qu'émergent des potentialités de manipulation étendues à la scénographie, à l'espace entier.

É.V. Certains marionnettistes considèrent la marionnette comme un personnage ; pour ma part, je crois que l'animation est un rapport aux choses. En tant que marionnettiste, c'est en ce sens que j'ai envie de développer mon travail. De ce point de vue la figure de la sœur Bellangère et des servantes que l'on incarne avec la marionnettiste Sarah Lascar de manière dissociée et passagère est très marionnettique. On ne voit pas Bellangère mais on entend sa voix en direct, on voit ces corps en robes noires qui rôdent autour des scènes, regardent par les fenêtres, elles sont à la fois des figures qui aident Ygraine mais aussi les servantes qui vont venir prendre l'enfant. Il en est de même pour le rôle d'Ygraine, joué par Julie Denisse en alternance avec Stéphanie Farison, qui même s'il nous apparaît ancré dans une action et incarné en chair et en os, n'est jamais traité de manière mimétique. Ce qui concerne aussi la scénographie: elle est animée parce qu'on propose au spectateur de vivre une expérience dans cet espace. Il y a une sorte de cohérence au niveau du fond et de la forme sur le plan de ce rapport au monde, qui se rapproche d'une conception animiste, dans le sens que Jérémy Damian donne à ce mot. Je pense que le fait d'animer met aussi à distance, permet de ne pas être dans l'incarnation et de créer plusieurs niveaux de lecture et de possibilités de sens. On a vraiment pris appui sur l'idée du rituel : chaque objet a une vie, il est animé.

A. C. Justement, dans le jeu d'Ygraine le traitement des adresses et des regards fait que ses répliques renvoient autant à son rapport avec Tintagiles qu'à son rapport avec ellemême.

É. V. Oui, car nous avons choisi de traiter chaque personnage comme une figure. La représentation réaliste, l'incarnation des personnages a souvent cet écueil de proposer une vision unilatérale. Dans ce cas, en tant que spectatrice, je préfère en rester à la lecture, car ce mode de représentation ferme toute réception sensible et personnelle. Maeterlinck combattait le surjeu des comédiens qui tuaient le poème et prônait un jeu distancié présent dans le théâtre de marionnettes. Cet objet ritualisé se construit et se comprend par la déambulation. On peut se dire que chaque figure participe à un ensemble. 
L'Enfant (4)

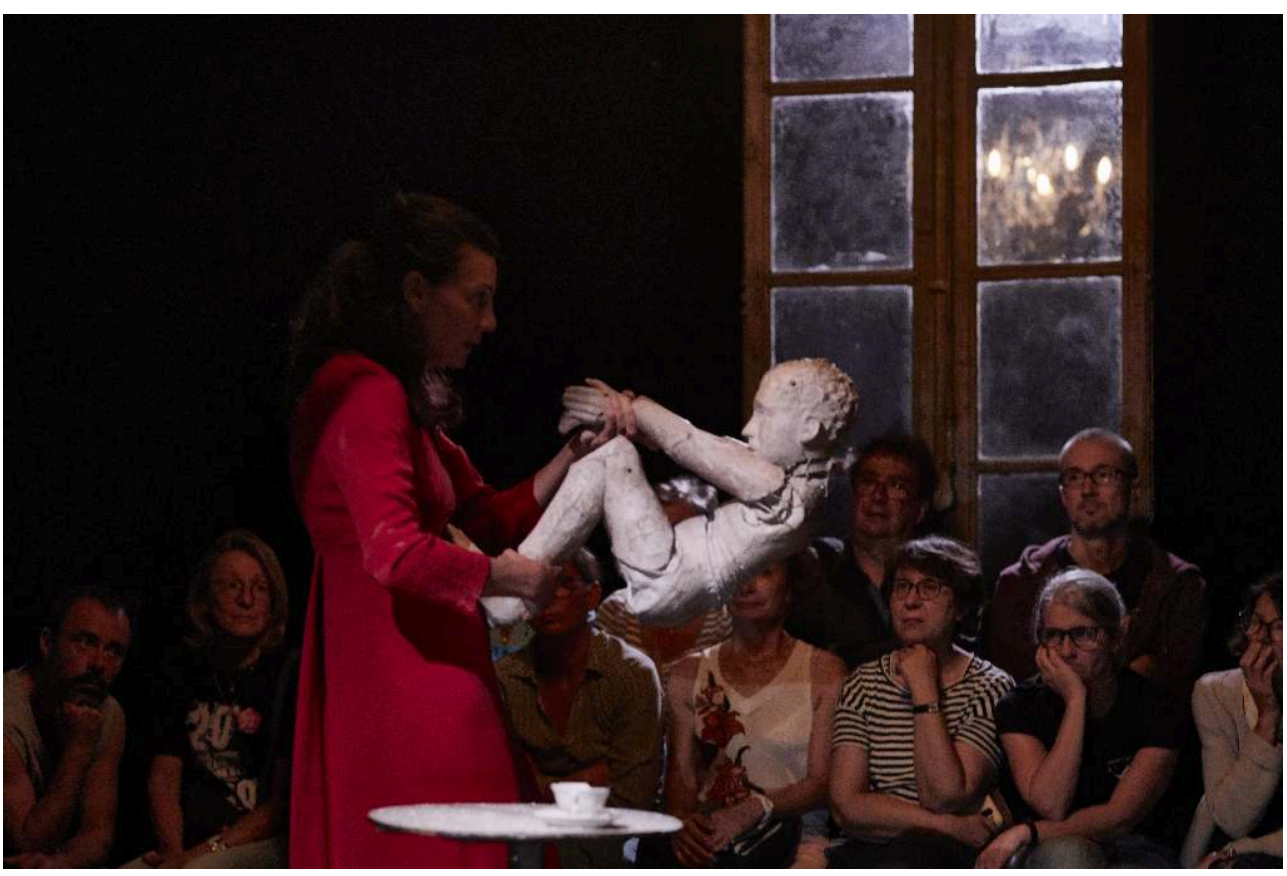

\section{La glace : éprouver, expérimenter}

A. C. J'aimerais qu'on revienne plus spécifiquement sur ton travail autour des matériaux fluides et de la glace - d'abord dans la genèse du projet, parce que si je ne me trompe tu avais travaillé avec une glaciologue, mais aussi au niveau dramaturgique, et du point de vue des contraintes technique spécifiques à ce spectacle.

É. V. Le point de départ a été les poèmes de Tarjei Vesaas qui parlent d'une nature assez tellurique - c'est la Norvège et il y a beaucoup de glace et les éléments naturels sont très présents. Chaque poème place la nature comme une métaphore de la condition humaine. Je voulais vraiment travailler sur cette idée d'impermanence, d'abord par le choix des matériaux : la glace, mais aussi le feu. Dans Impermanence une multitude de pieds d'âges différents en glace marchaient sur une plaque chauffante et se transformaient en vapeur, c'était un poème sur Hiroshima. On utilisait un congélateur et on travaillait par étapes : il suffisait de faire des moules en élastomère et de démouler. Pour Anywhere la construction a été plus compliquée, car la marionnette est articulée. De plus c'est une marionnette à fils où chaque crochet doit être précisément positionné, ce qui n'est pas facile dans une matière liquide au départ! Tout le corps est en plusieurs parties, avec une petite structure en corde à l'intérieur. C'est Hélène Barreau qui a construit la marionnette de glace d'cEdipe ; elle a dû trouver plein de petits bricolages au fur et à mesure des difficultés que l'on rencontrait: nos moules ont fui parce que c'était trop lourd, elle a dû tout refaire, ensuite la glace se fissurait avec les différences de température, les cordes rétrécissaient... On butait, dans un processus de création qui était court par rapport à la recherche et tout était nouveau pour nous. Et on ne pouvait pas téléphoner à un spécialiste de la glace ou à des gens qui avaient fait la même chose avant nous, car on ne connaissait personne qui avait fait ça. Ensuite j'ai rencontré la glaciologue Maurine Montagnat, nos échanges ont beaucoup nourri mon travail, on est d'ailleurs toujours en contact. Mais elle travaille dans des conditions très précises, en 
laboratoire, et ce n'est pas reproductible au théâtre - encore moins en tournée. Il a donc fallu bidouiller, bricoler. Il y avait aussi la question de la fonte qu'il fallait doser : il ne fallait pas que ça fonde trop - comme pour l'écran de plâtre à la fin de L'Enfant, parfois le plâtre tombe trop parce qu'il est trop humide ou au contraire il ne tombe pas assez. C'est un peu comme avec une marionnette. Parfois on commence un spectacle avec un story-board, par exemple on voudrait que la marionnette marche, même si elle n'est pas faite pour cela. Dans ce cas il vaut mieux changer d'idée et voir quelles sont les spécificités de cette marionnette, quel est son langage, sa façon de bouger et ensuite perfectionner la technique pour que le mouvement paraisse le plus naturel possible; cela ne sert à rien de chercher un autre langage avec cette même matière et de s'acharner, il vaut mieux suivre là où t'emmène la matière. Picasso dit " je ne cherche pas je trouve », cela nécessite beaucoup de travail, mais les trouvailles apparaissent quand on n'essaye plus de trouver mais que l'on suit ce qui se présente à nous.

Puis la logistique se met en place. Au début Anywhere c'était un stress permanent. Ensuite, on a pris le coup après avoir rencontré pleins de problèmes, maintenant Hélène Barreau a un rythme de fabrication, mise en glace, démoulage, assemblage très précis et si on a des imprévus elle a des solutions. Au niveau logistique c'est assez simple, par contre ça prend énormément de temps. On peut jouer facilement à l'international avec ce spectacle, dans ce cas on demande les congélateurs sur place. Sinon, quand on joue en France, on part avec nos quatre congélateurs. Voici comment se passe le planning: on arrive l'avant-veille du montage. Hélène Barreau, qui joue aussi dans Anywhere, construit la marionnette de glace. Moi, je m'occupe de l'écran de glace, je dispose les résistances chauffantes dans l'eau, on met tout au congélateur pendant toute la nuit. Puis le lendemain il faut y être pour démouler, articuler, ça prend quasiment la journée et le soir il faut tout relancer : après avoir joué on passe quasiment trois heures pour la mise en glace (mettre en place les structures, couler l'eau, serrer les serre-joints...). Et le lendemain on y est dès 9 heures pour préparer.

Pour L'Enfant j'avais envie que ce soit plus simple mais aussi plus en lien avec le jeu, et moins avec l'idée plastique. Dans ce spectacle, la technique est aussi importante que l'artistique. Je voulais créer un environnement vibrant, mais sans passer plus de temps dans la construction que sur le plateau, parce qu'après c'est très long de rôder le spectacle. Pour Anywhere il fallait quasiment six heures pour faire la construction tous les jours - et avec la glace on ne peut pas répéter plusieurs fois la scène car elle fond... Avec une grosse équipe et quelqu'un qui est spécialisé là-dedans c'est possible, mais quand ce sont les mêmes personnes qui font tout ce sont des processus assez fragiles. Mais finalement, c'est aussi cette fragilité qui donne de la force à ces propositions. Même aujourd'hui, après plus de 100 représentations, on est toujours sur le qui-vive pendant tout le déroulé du spectacle, cela nous met dans un état de présence obligatoire d'où émane une certaine tension sur scène. Quand on arrive dans les lieux qui ne sont pas habitués à ce genre de travail il faut bien prévenir, et anticiper. 
Anywhere (2)

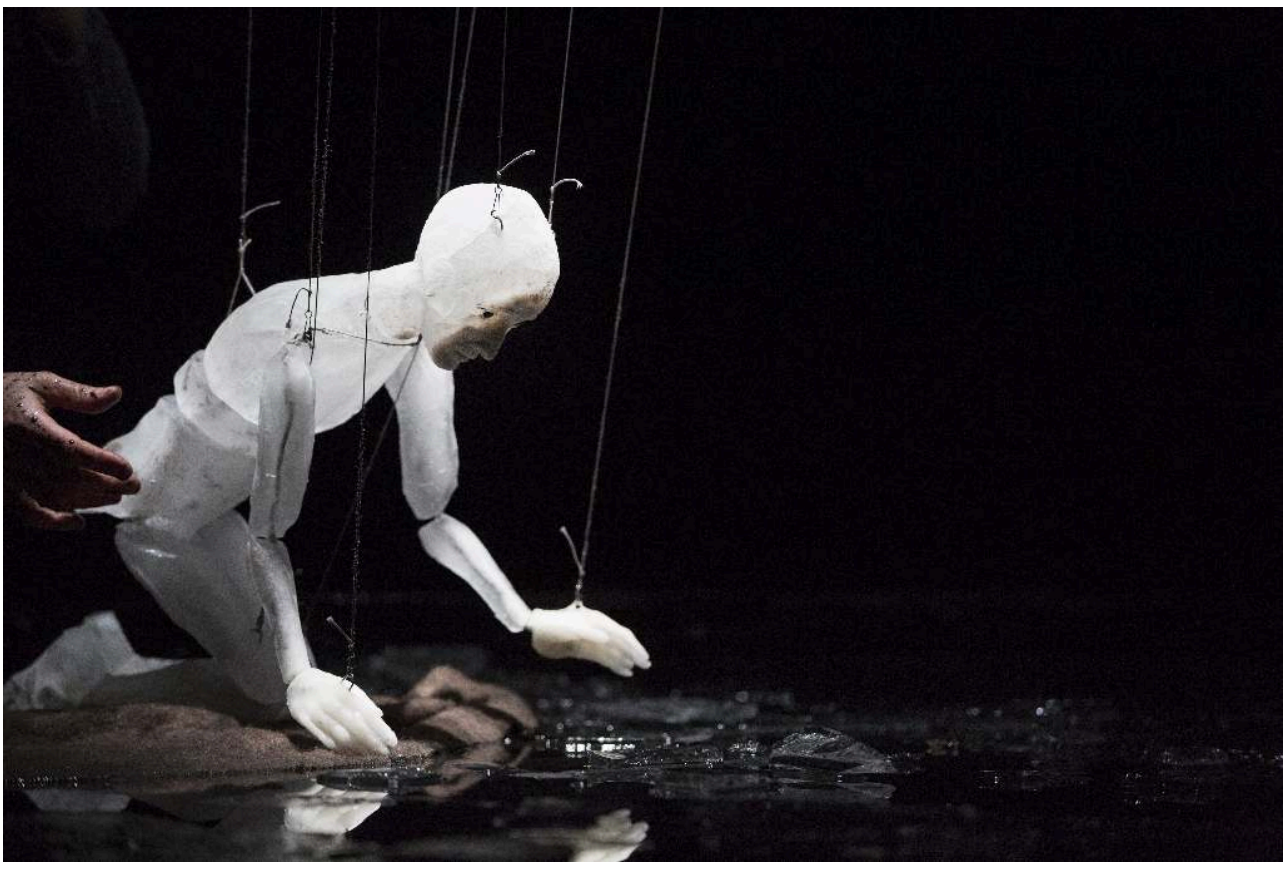

(c) Vincent Beaume

A. C. Quel a été l'impact des propriétés physiques de ce matériau-là sur la durée du spectacle, sur la dramaturgie...?

É. V. Pour Anywhere nous avons enchaîné les épreuves, c'était très compliqué. Mon idée de départ était une marionnette à fils : un Edipe en glace que je manipulais. Mais en fait c'était impossible : elle était beaucoup trop lourde $(5 \mathrm{~kg})$, elle glissait. Et c'est comme ça qu'on a eu l'idée de cette marionnette à longs fils et qu'on a commencé à suspendre le contrôle pour m'alléger le bras et supporter une partie du poids. Et avec Hélène Barreau on a construit la dramaturgie d'œEdipe et d'Antigone à partir de la contrainte technique. C'est comme ça qu'on a trouvé la distance entre sa fille et lui : il y a la manipulatrice à distance, mais je peux le porter, le pousser - avec des effets de pendule - et il peut aussi vivre tout seul. C'était la découverte d'une technique de manipulation liée à la contrainte technique. Sinon on aurait pu s'acharner pendant des heures et rater, alors que là on a pu prendre du recul, essayer et faire des découvertes. 
Anywhere (3)

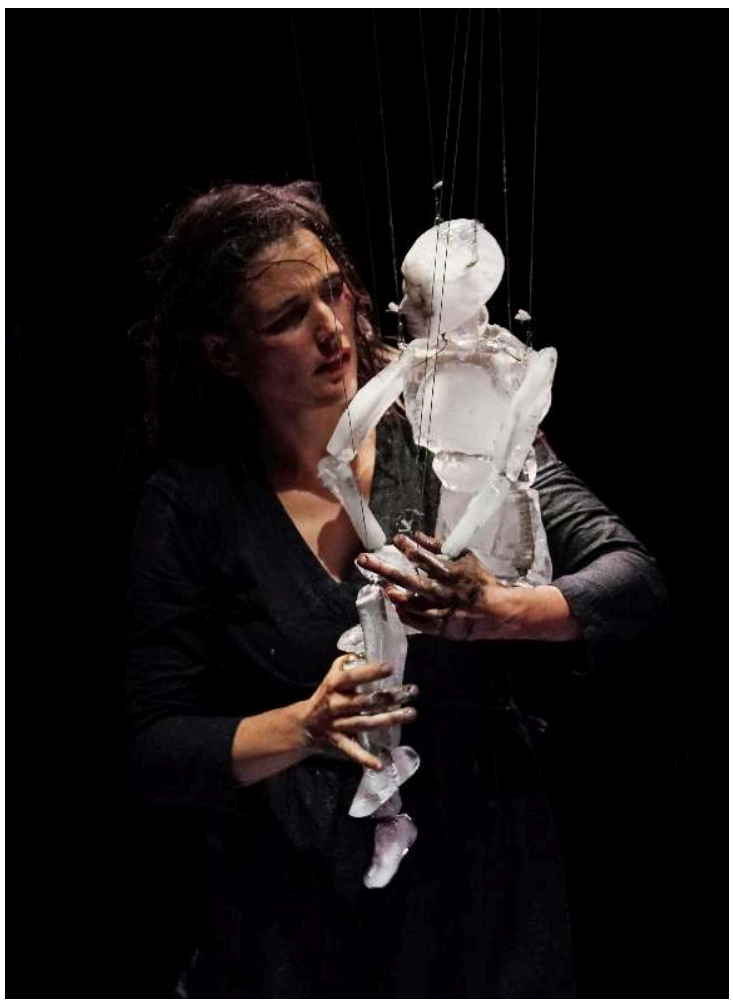

A. C. Tout à I'heure tu utilisais le mot "éprouver », c'est aussi l'idée ? II y a l'idée de se confronter aux matériaux, de se mettre à l'épreuve...

É. V. Oui c'est ça, la découverte des matériaux est une initiation. Anywhere tout particulièrement: tu passes par de gros moments d'incertitude mais c'est aussi un défi à relever, et une fois que l'objet est à peu près là il y a la question de la manière dont tu arrives dans les théâtres, le temps de montage, comment tu arrives petit à petit à rôder ton objet. Cela change aussi les choses pour les lieux d'accueil : la glace prend de la place, il faut des congélateurs, un atelier. Certains lieux comme le TJP (Théâtre Jeune Public - CDN Grand Est) comprennent ça très bien.

\section{Seuils}

A. C. Comment se passe le stockage? Que deviennent les matériaux de tes précédents spectacles?

É. V. J'ai un lieu de stockage qui est assez plein. Après, je ne suis pas tournée vers le passé : Impermanence ne tourne plus et je me dis qu'il faut recycler. Mais ça prend de la place oui : on stocke les congélateurs... C'est un travail qui ne peut pas être minimaliste et on retrouve d'ailleurs ces questions dans le montage, le démontage.

A. C. II existe des pratiques de récupération de matériaux et d'objets dans le champ des arts de la marionnette. Est-ce qu'il t'arrive de travailler de cette manière?

É. V. Non, je ne travaille pas comme ça. Dans L'Enfant il y a une marionnette en papier, mais c'est du papier qu'on a acheté. Je n'avais pas envie de travailler la résine, le papier me paraissait plus organique. Je suis plutôt dans une recherche de l'organique que dans une pratique de la récupération, en fait. Dans L'Enfant il y a des pierres, des vrais os que je suis allée chercher dans la montagne, des objets un peu 
" chargés ". Mais c'est tout : ce sont des vieilles fenêtres mais cela reste du décor, le bois est du contreplaqué qu'on a acheté. Dans la temporalité de la création c'est compliqué de mettre une annonce pour récupérer du contreplaqué, il y a aussi des contraintes de poids, de dimensions...

A. C. As-tu eu des expériences hors théâtre de type exposition, installation ou déambulation? Aimerais-tu aller dans cette direction?

É. V. J'ai fait des installations, avec des photos notamment. Et je vais reprendre mon solo Traversées avec la marionnettiste Kristina Dementeva qui va reprendre mon rôle, pour créer une forme en extérieure qui puisse se jouer en forêt, ou dans d'autres cadres naturels et donner un nouveau souffle à ce spectacle qui a dix ans.

A. C. Est-ce que la question du seuil, qu'on devine dans le nom du Théâtre de l'Entrouvert et qu'on retrouve dans tes spectacles - si on pense ne serait-ce qu'aux portes dans L'Enfant pourrait être un fil conducteur de ton travail ?

É. V. Le nom de la compagnie L'Entrouvert vient d'une phrase de René Char qui parle de l'entrouvert comme d'un " lieu de partage entre l'ombre et la lumière ». La marionnette nous situe à la frontière du visible et de l'invisible, de la présence et de l'absence, c'est un langage du passage.

Les matériaux en transformation sont aussi des seuils, des moments de déséquilibre, les cadres de portes sont présents concrètement dans la scénographie, et les textes de Maeterlinck ne parlent que de seuils. À travers ces différents états de matière, ces scénographies, le spectateur vit ces états de basculement qui sont des états de présence. Ces moments de passage, nous placent aussi dans un temps cyclique. Ce sont des territoires qui ne peuvent être limités à une narration mais nous devons les appréhender comme une expérience à vivre. Même dans L'Enfant, le texte n'est pas un support narratif, il reste une énigme. Je ne cherche jamais à expliquer mais au contraire à ouvrir des pistes.

\section{NOTES}

1. Pour lequel elle a été accompagnée par le Vélo théâtre (Apt).

2. Site internet de la compagnie : http://lentrouvert.com/ (consulté le 25/10/2019).

3. Spectacle lauréat du Festival international d'Ostrava 2017 et du prix Henri Bauchau 2018.

4. Centre régional des arts du cirque de Lomme - Lille.

5. Hélène Guenin (dir.), Sublime, les tremblements du monde, Éditions du Centre Pompidou-Metz, 2016.

6. Jérémy Damian, « Weird animisms », in Revue Corps-Objet-Image ${ }^{\circ} 3$, Ré-animation, Strasbourg, TJP Éditions, mars 2018, p. 5-12. Également accessible en ligne : https://static1.squarespace.com/ static/542cf50be4b0b0eacb4ab721/t/58e3a79e893fc01307513bdb/1491314590794/RevueCOI-03Weird+Animisms.pdf (consulté le 16/04/20).

7. Ibid., p. 98-99.

8. Yoann Moreau, Vivre avec les catastrophes, Paris, PUF, 2017.

9. Georges Didi-Huberman, Soulèvements, avec des essais de Nicole Brenez, Judith Butler, MarieJosé Mondzain, Antonio Negri et Jacques Rancière, Paris, Gallimard/Jeu de Paume, 2016. 
10. Marie-Louise von Franz, La Femme dans les contes de fées, Paris, Éditions du Dauphin, 1999.

INDEX

Mots-clés : matière, marionnette, Vigneron (Élise) 